\title{
Opinion Dynamics of Social-Similarity-Based Hegselmann-Krause Model
}

\author{
Xi Chen,, Xiao Zhang, ${ }^{1,2}$ Yong Xie, ${ }^{1,2}$ and Wei Li ${ }^{1,2}$ \\ ${ }^{1}$ School of Automation, Huazhong University of Science and Technology, Wuhan 430074, China \\ ${ }^{2}$ Key Laboratory of Ministry of Education for Image Processing and Intelligent Control of China, Wuhan 430074, China \\ Correspondence should be addressed to Wei Li; liwei0828@mail.hust.edu.cn
}

Received 27 April 2017; Revised 26 September 2017; Accepted 26 October 2017; Published 5 December 2017

Academic Editor: Sergio Gómez

Copyright (c) $2017 \mathrm{Xi}$ Chen et al. This is an open access article distributed under the Creative Commons Attribution License, which permits unrestricted use, distribution, and reproduction in any medium, provided the original work is properly cited.

\begin{abstract}
The existing opinion dynamics models mainly concentrate on the impact of opinions on other opinions and ignore the effect of the social similarity between individuals. Social similarity between an individual and their neighbors will also affect their opinions in real life. Therefore, an opinion evolution model considering social similarity (social-similarity-based HK model, SSHK model for short) is introduced in this paper. Social similarity is calculated using individual properties and is used to measure the social relationship between individuals. By considering the joint effect of confidence bounds and social similarity in this model, the role of neighbors' selection is changed significantly in the process of the evolution of opinions. Numerical results demonstrate that the new model can not only obtain the salient features of the opinion result, namely, fragmentation, polarization, and consensus, but also achieve consensus more easily under the appropriate similarity threshold. In addition, the improved model with heterogeneous and homogeneous confidence bounds and similarity thresholds are also discussed. We found that the improved heterogeneous SSHK model could acquire opinion consensus results more easily than the homogeneous SSHK model and the classical models when the confidence bound was related to the similarity threshold. This finding provides a new way of thinking and a theoretical basis for the guidance of public opinion in real life.
\end{abstract}

\section{Introduction}

Over the past decades, opinion dynamics as a special type of complex human behavior has attracted a great deal of interest from researchers in different scientific fields [1]. Opinion dynamics, including opinion formation, spread, and evolution, has great influence on politics [2], economics [3], and society [4]. Existing studies have examined the inherent mechanism of the spread of opinion by establishing several opinion evolution models to forecast or influence public opinion [5].

In the existing study of opinion dynamics, models can be divided into discrete opinion models and continuous models $[6,7]$. Continuous opinion dynamics models are considered more suitable for representing complex opinions [7]. The most popular continuous opinion spreading models are the Deffuant model and the Hegselmann-Krause (HK) model [8-10], also known as bounded confidence models. In these models, the confidence bound (also known as opinion threshold or tolerance) is the main factor that influences opinion consensus and drives stabilization [11]. Individuals choose to communicate with neighbors according to the bounded confidence principle, and they only communicate with neighbors if their opinion distance is below the opinion threshold, that is, the confidence bound, and then may change their opinion $[12,13]$. Liang et al. found that a critical confidence bound value (0.5) is unaffected by network topology; moreover, the opinion of the whole system reaches a consensus when the confidence bound of all agents is above the critical value [13-15]. On the contrary, when below the threshold, it is difficult for the opinion to reach consensus. In the Deffuant model, every agent can only communicate with one of his neighbors at each time step, whereas every agent can communicate with all his neighbors in the HK model, which serves to alter the present situation of the social network $[16,17]$. The main outcomes of opinion evolution are fragmentation, polarization, and consensus. The aim of the present study is to determine how to make an opinion reach 
consensus. A significant amount of research has improved the classical HK model by combining it with reality. Liang et al. [13] proposed the use of a random or exponential distribution to represent the confidence bound. Different attributes or environments have disparate impacts on the confidence bound of agents. Fu et al. [14] proposed a modified Hegselmann-Krause model (MHK model) that considers the self-belief parameter, indicating the persistence of an individual's opinion and the persistence of other agents' opinion. It turns out that the self-belief parameter has great influence on opinion consensus.

However, the classical HK model and these improved HK models only consider the influence of neighbors' opinions on an individual's opinion in the process of communication. Many studies have proved that the change in an individual's opinion is also influenced by the social attributes of other agents [18-20]. Flache and Mäs [21] studied the effect of demographic fault lines and the timing of contacts on team cohesion, and their model is based on the assumption that the influence of agent $i$ on agent $j$ is not directly correlated with opinion, but with demographic similarity. Mäs et al. [22] found that crisscrossing actors could help in overcoming conflicts even in groups with strong demographic fault lines, and the time required for groups with a strong personality to overcome polarization is shorter than those with a weak personality. Taking this factor into account, the concept of social influence of individuals, namely, social power, is introduced into the opinion evolution model. Social power is determined by some features of network topology such as node degree and betweenness centrality and follows a power-law distribution. The simulation result shows that appropriate social power distribution can enable opinions to converge easily $[19,20]$. However, individuals in the social network are so complex that the use of network topology to represent social power is far from enough. Everyone has different social attributes and resources and can be affected by different influences. In models with similarity-biased influence, only sufficiently similar individuals can interact with each other to reduce opinion differences, and similarity threshold depends on additional psychological mechanisms (e.g., social identity, self-confidence) [23]. Anyone can have the same social power on all the other neighbors, ignoring the interaction between two individuals.

This paper combines the joint influence of neighbors' opinions and social attributes on an individual's opinion. Likewise, the opinion of some agents outside the confidence bound, which can also communicate, is considered. In Section 2, a social-similarity-based Hegselmann-Krause model (SSHK) is proposed, by introducing social similarity in selecting communicating neighbors. The simulation result and analysis of the homogeneous (the confidence bound and similarity threshold show equality) and heterogeneous models (the confidence bound and similarity threshold show inequality) are presented in Section 3. Section 4 presents a discussion and the conclusion.

\section{Method}

2.1. Similarity-Based Social Relationship. The intimacy of a social relationship between individuals can be described by their social similarity. "Birds of a feather flock together" is a saying which means that people of the same kind show stronger interpersonal attraction. Interpersonal attraction is a state of mutual dependence and is a positive form of relationship. One of the most important principles of interpersonal attraction is the similarity principle [20]. People tend to make friends with and trust others who share the same hobbies or the same social status as themselves [20]. Thus, similarity based on an individual's social attributes is introduced in this paper to describe social relationship. The existing agent-based research on opinion evolution always treats individuals as a simple node. Different social attributes of people, such as age, gender, education level, wealth, and geographical location, are relatively less studied in natural science. These attributes are an integral part of the description of unique individuals that cannot be neglected. Thus, this paper introduces social attributes to the classical bounded confidence model (HK model) to identify individuals.

The possible values of each social attribute are limited, and the range of values of each social attribute is summarized according to empirical analysis. To facilitate the modeling calculation, each attribute value is simply quantified. There are two kinds of quantitative methods-one is a certain number of discrete values corresponding to the value of the property, and the other quantifies social attributes as a range of continuous values, and the social attributes themselves are represented by a range of values. It is obvious that the latter method has better representation effects, so that continuous values are used to represent individual attributes. Each agent will be assigned attributes forming an attribute set, including age, gender, education level, economic status, and geographic location according to the fifth population census data of China. For example, age is a nonnegative integer represented by a number between 0 and 100, and education level can be expressed as a float number between 0 and 1, where 0 stands for the lowest education level (illiteracy) and 1 stands for the highest education level. Gender can be specified as male or female, and geographic location can be represented by the latitude and longitude of an individual's residence.

Specifically, agent $i$ can be expressed as a set of values $\left\{\operatorname{attr}_{i, 1}, \operatorname{attr}_{i, 2}, \ldots, \operatorname{attr}_{i, m}\right\}$. Moreover, it can be seen as a node in the $m$-dimension of social attribute space.

The intimacy of the social relationship between individuals can be described by their social similarity $S_{i, j}$. Similarity between node $i$ and node $j\left(S_{i, j}\right)$ is computed based on the individual attribute vector. According to different types of values, $S_{i, j}$ is classified into identical similarity $\left(S_{i j}^{1}\right)$, degree similarity $\left(S_{i j}^{2}\right)$, and reverse similarity $\left(S_{i j}^{3}\right) . S_{i j}^{1}$ is defined for attributes with only two values. Here, only gender is included. $S_{i j}^{2}$ is defined for the attribute with a float number. Here, age similarity $\left(S_{i j}^{\text {age }}\right)$, education similarity $\left(S_{i j}^{\text {edu }}\right)$, and economic similarity $\left(S_{i j}^{\text {eco }}\right)$ are included. $S_{i j}^{3}$ is defined to measure the geographical position. It is inversely proportional to the distance between the two nodes, which is based on the Hamming distance. Details of the above definitions can be found in [24]. 
By combining $S_{i j}^{1}, S_{i j}^{2}$, and $S_{i j}^{3}, S_{i j}$ is calculated as

$$
S_{i j}=\left[W^{1}, W^{2}, W^{3}\right] \cdot\left[S_{i j}^{1}, S_{i j}^{2}, S_{i j}^{3}\right]^{T}
$$

where $W^{1}, W^{2}, W^{3}$ are the weights of $S_{i j}^{1}, S_{i j}^{2}$, and $S_{i j}^{3}$, respectively, as specified by experts. For the convenience of calculation, the values of similarity are normalized within $[0,1]$. In Section 3, social attributes are simplified in this version and two attributes of great importance on social relationships in real life (geographic position and education level) are considered here in order to facilitate the analysis; for example, education level in developed areas will be generally higher than that in developing areas. Geographic position belongs to $S_{i j}^{3}$ and education level belongs to $S_{i j}^{2}$, and the two attributes are closely related; for example, education level in developed areas will be generally higher than that in developing areas. Therefore, $W^{1}$ is set to a value of 0 , and $W^{2}, W^{3}$ are set to a level of 0.5 , respectively.

2.2. Model. In the classical $\mathrm{HK}$ model, each agent selects another agent whose opinion is limited in the confidence bound as communication neighbors $[12,25]$. An agent then averages his and his neighbors' opinion as his new opinion. Fu [14] proposed an MHK model that considers self-confidence based on the classical $\mathrm{HK}$ model. He uses a self-belief parameter $\lambda$ to represent the agent's degree of retaining his own opinion. Thus, $1-\lambda$ is on behalf of the agent's degree of considering his neighbors' opinion. Two extreme situations are $\lambda=1$, which represents an extremely stubborn agent who only believes in his own opinions, and $\lambda=0$, which describes a fully open-minded agent who only has faith in his neighbors' opinions. The exchange rules of the MHK model are as follows:

$$
\begin{aligned}
& x_{i}(t+1) \\
& \quad= \begin{cases}\lambda_{i} x_{i}(t)+\frac{\left(1-\lambda_{i}\right)}{\left|\bar{N}_{i}(t)\right|} \sum_{j \in N_{i}} x_{j}(t), & \bar{N}_{i}(t) \neq \phi \\
x_{i}(t), & \bar{N}_{i}(t)=\phi,\end{cases}
\end{aligned}
$$

where the communication neighbor of agent $i$ is $\bar{N}_{i}(t)=$ $\left\{j|| x_{i}(t)-x_{j}(t) \mid \leq \varepsilon_{i}, j \neq i\right\}$, where $\varepsilon_{i}$ is the confidence bound and $\lambda_{i}$ is his self-belief parameter. $\lambda$ has an influence on opinion convergence, which means that a small $\lambda$ corresponds to a short converging time that a social system requires.

Based on the MHK model, this paper proposes the SSHK model, which introduces social similarity to represent the social relationship between an agent and his neighbors. An agent's neighbors can be selected by their social similarity and confidence bound. The SSHK model has $N$ agents. Each agent holds an opinion $x_{i}(t) \in[0,1]$ and has a social attribute set. At each time step, each agent $i$ changes his opinion according to formula (2). The selection of communication neighbors $\bar{N}_{i}(t)$ is discussed in the following analysis.

The classical bounded confidence model shows that when communicating with each other, the agents may be convinced by their neighbors who share a similar opinion with him, and every one may change his opinion toward his neighbors. However, the agent also changes his opinion toward his close or reliable neighbors regardless of his own opinion, indicating that people's dependence on social relations and the objective judgment of opinion in communication matter in the process of opinion exchange. To consider the above two situations simultaneously, social similarity between agents is introduced to extend the confidence bound. A social similarity threshold $s_{\text {threshold }}$ is set here to represent social relations or trust degree between agents. An agent considers the confidence bound $\varepsilon$ and similarity threshold $s_{\text {threshold }}$ when selecting communication neighbors.

In the process of neighbor selection, two constraints are set up simultaneously: $s_{i, j}<s_{\text {threshold }} \&\left|x_{i}(t)-x_{j}(t)\right|<\varepsilon$. The selection rules are more demanding than the classical model, which is not realistic. There is often a case where some agents who share a strong social relationship or high social similarity have contradicting opinions, and they may also communicate with each other and exchange opinions. Thus, the weighting methods $w_{s}$ and $1-w_{s}$ are used to integrate the weights of social similarity and the confidence bound simultaneously, to represent different influences $\left(W_{s} \in[0,1]\right)$. Thus, the set of neighbors of agent $i$ can be calculated as follows:

$$
\begin{gathered}
\bar{N}_{i}(t)=\left\{j \mid\left[w_{s} *\left(S_{i j}-S_{\text {threshold }}\right)+\left(1-w_{s}\right)\right.\right. \\
\left.\left.*\left(\left|X_{i}(t)-X_{j}(t)\right|-\varepsilon_{i}\right)\right]<0, j \neq i\right\} .
\end{gathered}
$$

This paper ignores the influence of network topology and assumes that the network is fully connected. Therefore, each agent can communicate with all other agents in the system. However, every agent can only communicate with the neighbors calculated by formula (3) that are constrained by $s_{\text {threshold }}$ and $\varepsilon$. Social similarity is different from the self-belief parameter. Self-belief is the description of the agent's own personal attribute and is the inherent nature of an individual. Obviously, it does not change when facing different neighbors. Since social similarity is used to describe the relation and interaction between an individual and his neighbors, the social similarity of the same agent changes when facing different neighbors.

\section{Simulation Analysis and Result}

The SSHK model is studied using agent-based modeling and simulation. The simulation result is averaged 100 times. The system assumes a fully connected network of $N=1000$. Opinion is a continuous value between 0 and 1 , and the initial opinion of each individual follows a uniform distribution [14]. The agents are divided into three categories: a shaky agent $(\lambda=0.05)$ who is easily affected by neighbors' opinions, a mild agent $(\lambda=0.4)$ who considers both his own and his neighbors' opinions, and a stubborn agent $(\lambda=0.9)$ who adheres to his own opinions and the impact of other agents is weak. In the following research, two social attributes (geographic position and education level) are chosen in order to facilitate analysis and every agent has a two-dimensional attribute $\left\{\operatorname{attr}_{i, 1}, \operatorname{attr}_{i, 2}\right\}$, such as geographic position and education level. The similarity between agents can be calculated by formula (1). 
Steady state is defined when the opinion of all agents no longer changes; that is, the system is stable [13]. One important observation indicator of opinion dynamics research is the final number of opinions, Cluster $F$. When reaching steady state, $F$ shows three different opinion states of fragmentation, polarization, and consensus. Fragmentation refers to a situation where the whole population is divided into a lot of subgroups and the opinions of these subgroups are different. Polarization refers to a situation where the whole population is divided into several subgroups, and the opinions of these subgroups are different. If the difference between two opinions is less than $10^{-4}$, they can be regarded as the same opinion or an opinion cluster. Another observation indicator is simulation time $T$-the time that a system requires to reach the steady state.

Homogeneous and heterogeneous models are discussed in the following sections. A homogeneous model means that the confidence bound and similarity threshold are equal. A heterogeneous model means that the confidence bound and similarity threshold are unequal.

3.1. Homogeneous SSHK Model. The homogeneous model is studied in this section. The confidence bound is neglected to analyze the impact of social similarity on opinion dynamics. The influences of the confidence bound and social similarity threshold are compared when they work simultaneously. The SSHK model is verified by comparing it with the classical HK and MHK model.

3.1.1. The Influence of Social Similarity. First, we assume that $W_{s}=1$; that is, we only study the effect of social similarity. Different opinion evolution results are obtained by changing the similarity from 0.01 to 0.1 . A few representative results are shown in Figure 1.

Figure 1 illustrates opinion evolution under different conditions of social similarity. From the result, it can be seen that opinion polarization cannot be achieved. Opinion becomes fragmented when the social similarity threshold is small $\left(s_{\text {threshold }}=0.01\right)$. As social similarity increases, most opinions can converge to a single opinion while others are in a decentralized state $\left(s_{\text {threshold }}=0.03\right)$. When the similarity is large enough $\left(s_{\text {threshold }}=0.05\right.$ and $s_{\text {threshold }}=$ 0.1 ), opinion follows the rule of gradually converging and finally coming to consensus. Thus, social similarity influences opinion dynamics to achieve the consensual stability of opinions with the increase in similarity threshold. This finding is attributed to the similarity threshold, which does not directly change opinions during communication, and is only used to select communication neighbors. Similarity threshold has an indirect impact on opinions, whereas the confidence bound has a direct influence on opinions. Moreover, the similarity threshold of several representative values of $0.01,0.03,0.05$, and 0.1 can be obtained.

3.1.2. The Mutual Influence of Similarity and Confidence Bound. The reasonable value of $w_{s}$ and the influences of social similarity thresholds as well as confidence bounds are compared in this section.
The influence of $w_{s}$ is analyzed based on the differences between the confidence bound and different similarity threshold. By simulating the HK, MHK, and SSHK models, the following representative confidence bound values can be obtained: $\varepsilon=0.05$ (opinion fragmentation), $\varepsilon=0.2$ (opinion polarization), and $\varepsilon=0.3$ (opinion consensus). The values are used in the following simulation. Since opinion objectives affect relationships between people more than social similarities, extreme similarity weights are ignored in the model $\left(w_{s}=0.9 \sim 1\right)$.

Based on the assumption that most individuals are generally objective, we assume that the weight of the confidence bound is greater than the weight of the similarity $\left(w_{s}=0.3\right.$ and $s_{\text {threshold }}=0.1$ ); specifically, the confidence bound and similarity play a role in opinion evolution simultaneously. We analyze the effect of weights under different confidence bounds and similarity thresholds and obtain the most appropriate weight value. Since the sum of the weight of the confidence bound and similarity is 1 , we only discuss the weight of the similarity and analyze the influence of the different weights on opinion evolution. Figure 2 shows the effect of the similarity threshold on the opinion numbers under several different similarity thresholds.

The similarity threshold is small $\left(S_{\text {threshold }}<0.1\right)$. When the weight of similarity is small $\left(W_{s}<0.3\right)$, that is, when the weight of the confidence bound is large, the final state of opinion is completely determined by the confidence bound. When $w_{s}>0.6$ and the confidence bound is small $(\varepsilon=0.05)$, the simulation result is opinion fragmentation. When the confidence bound is moderate $(\varepsilon=0.2)$, opinion polarization can be obtained, and a large enough trust radius $(\varepsilon=0.3)$ can ensure that some of the views will achieve convergence.

The similarity threshold is large $\left(S_{\text {threshold }}>0.1\right)$. An opinion reaches consensus when $i$ is very small, and the stability of the opinion increases with the increase in the similarity threshold. When $W_{s}>0.8$, irrespective of the value of $\varepsilon$, the opinion will not achieve polarization and fragmentation. Therefore, the opinion is not entirely determined by the similarity, and the confidence bound is still relevant. It is noteworthy that when $W_{s}>0.8$, an opinion can achieve consensus even when the confidence bound is very small; this is because the SSHK model increases the communication opportunities of agents outside the confidence bound, making it easier for the whole system to achieve consensus.

It can be seen that when the weight of similarity is at the intermediate value $\left(W_{s}=0.3 \sim 0.7\right)$, the confidence bound and the similarity have joint effects, and the state of opinion is relatively random; for example, when $w_{s}=$ $0.3, s_{\text {threshold }}=0.03$, and $\varepsilon=0.3$, the opinion achieves polarization rather than consensus and fragmentation. As can be seen from Figure 2, when the weight of similarity is between 0.3 and 0.7 , the confidence bound and the similarity work together. In theory, when the weight of the confidence bound is large, the opinion is mainly determined by the confidence bound, and when the weight of the similarity is large, the opinion is mainly determined by the similarity. Moreover, the appropriate weight can make these two factors work simultaneously. Taking into account the fact that the 

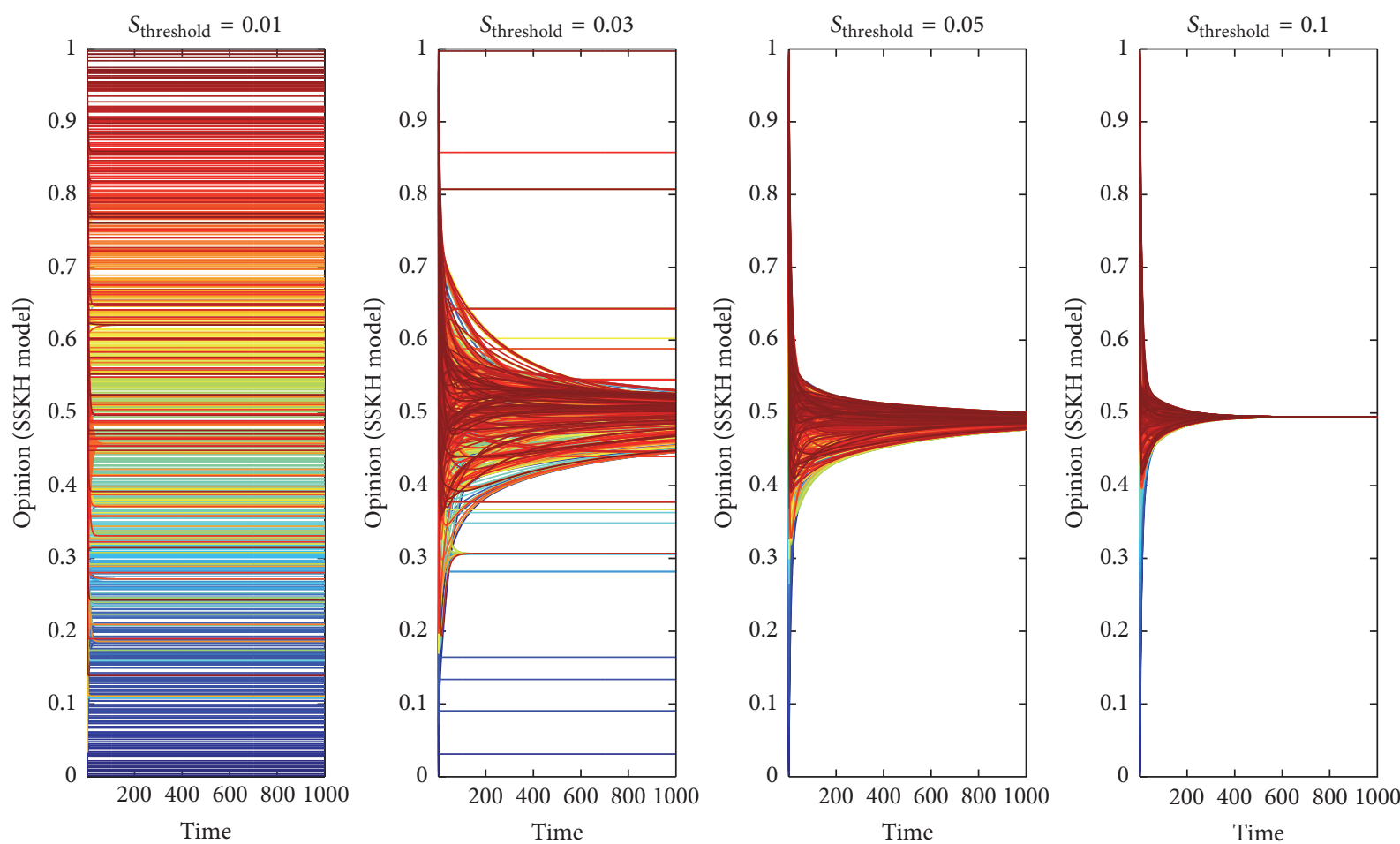

FIGURE 1: Opinion evolution results of the SSHK model by changing the social similarity with different $s_{\text {threshold }}\left(W_{s}=1\right)$.
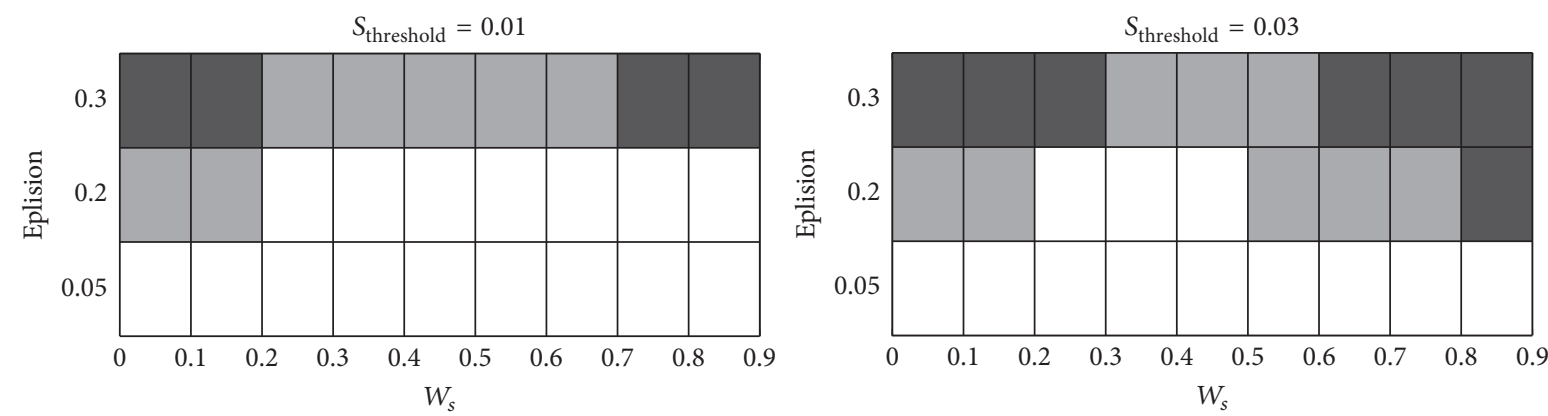

$S_{\text {threshold }}=0.1$
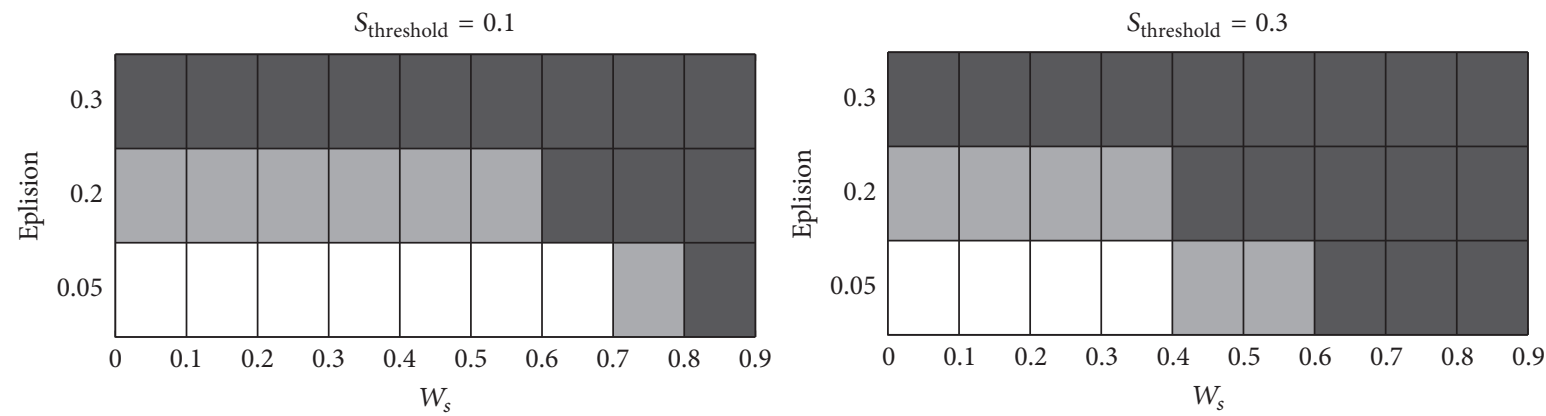

Figure 2: Opinion evolution result under the mutual influence of social similarity and the confidence bound of different $w_{s}$ (the dark gray, white, and light gray areas represent opinion consensus, opinion polarization, and opinion fragmentation, resp.).

confidence bound should play the dominant role in opinion evolution in the following study, we still choose the value of the previous hypothesis; that is, $w_{s}=0.3$.

It is noteworthy that when $w_{s}$ is not very small and $s_{\text {threshold }}$ is large, even if the confidence bound is small, the opinion can reach consensus, as shown in the black region in Figure 2. Since the model increases the exchange opportunities of the agents whose opinion difference is larger than the confidence bound, it is easier for the whole system to achieve consensus.

The influence of the similarity threshold in different confidence bounds is also studied. The results are shown in Figure 3.

In Figure 3(a), the final opinion number gradually decreases $(\varepsilon=0.05)$ with the increase in $s_{\text {threshold }}$. The opinion state changes from fragmentation to polarization with the 

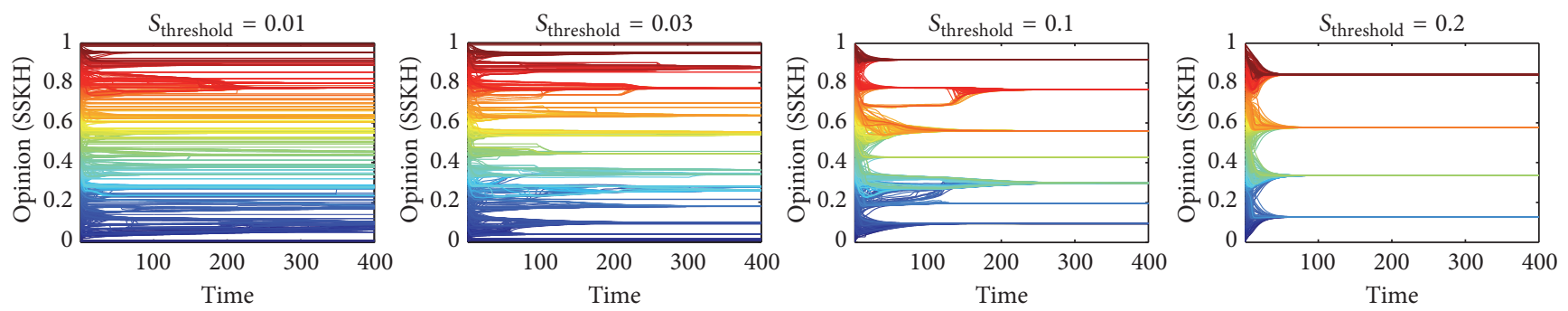

(a) $\varepsilon=0.05$
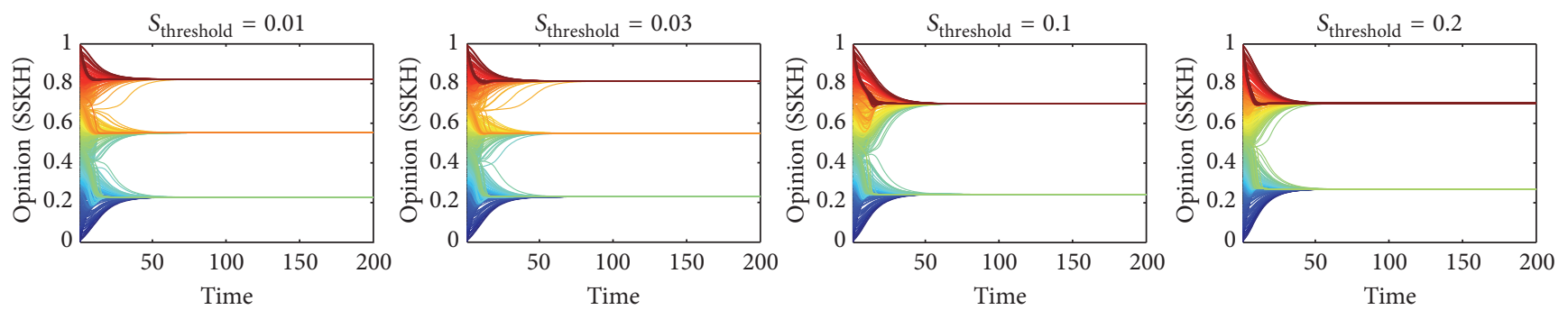

(b) $\varepsilon=0.2$
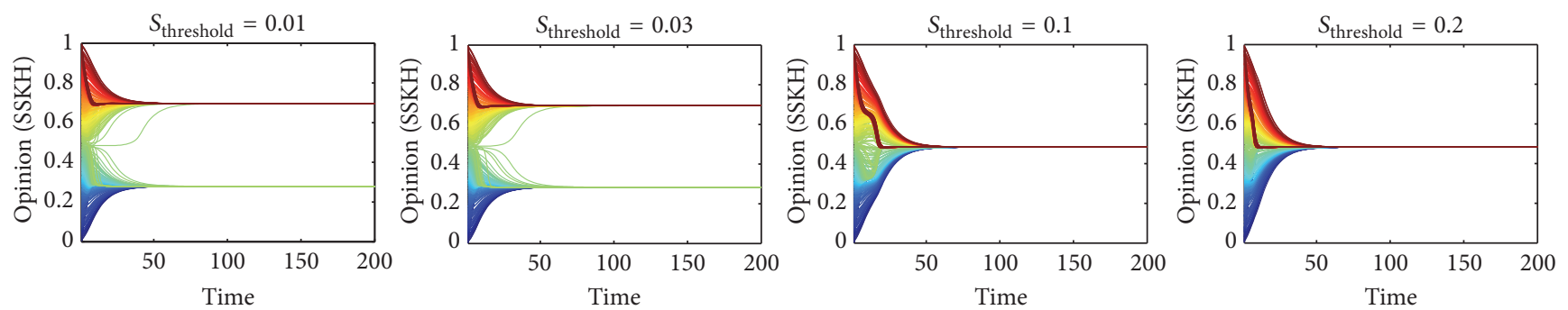

(c) $\varepsilon=0.3$

FIGURE 3: Influence of the similarity threshold on opinion evolution results under different confidence bounds.

increase in $s_{\text {threshold }}(\varepsilon=0.2)$ (Figure 3(b)). The opinion state changes from polarization to consensus with the increase in $s_{\text {threshold }}(\varepsilon=0.3)$ (Figure $\left.3(\mathrm{c})\right)$. The opinion result of fragmentation-polarization-consensus with the joint effects of social similarity and the confidence bound can be obtained by changing $s_{\text {threshold }}$. Therefore, social similarity also affects opinion dynamics.

3.1.3. Model Validation. To verify the feasibility of the SSHK model, we compare it with that of the classical HK and MHK models. The opinion distributions of the three models with different confidence bounds are shown in Figure 4.

Figure 4 demonstrates that the final opinion states of the three models are the same (i.e., fragmentation, polarization, or consensus). The main feature of the opinion result of the three models is opinion fragmentation when $\varepsilon=0.05$, polarization when $\varepsilon=0.2$, and consensus when $\varepsilon=0.3$. Regardless of how $\varepsilon$ changes, the classical HK model requires the shortest time to reach consensus, and the SSHK model requires the longest time to achieve a stable state when $\varepsilon$ is small $(\varepsilon=0.05)$. The MHK model shows exactly the same result as the $\mathrm{HK}$ model, whereas the opinion result of the SSHK model is different from that of the MHK model. The differences are discussed below. Figure 5 shows the difference between the SSHK model $\left(s_{\text {threshold }}=0.1,0.2\right)$ and the MHK model when $\varepsilon=0.05$. Table 1 compares the stable time $(T)$ and the opinion cluster number $(F)$ and the relative size of the largest cluster $N_{\max }$.

In Figure 5(a) of the MHK model, the agent number of every opinion cluster is almost the same, which is the same as that of Figure 5(c). However, in Figure 5(b) of the SSHK model, some opinion clusters contain a significantly larger agent number, whereas others only have a few agents. The agent number of the maximum opinion cluster is almost the same in the two models (Table 1). When the similarity threshold increases from 0.1 to 0.2 , the opinion number of the SSHK model is significantly less than the opinion number of the MHK model. The increase in the similarity threshold enables the agent outside the confidence bound to communicate with others. The opinion easily reaches consensus.

Table 1 shows that the stable time $T$ of the SSHK model with $s_{\text {threshold }}=0.1$ is longer than that of the MHK model. By contrast, the stable time of the SSHK model with $s_{\text {threshold }}=$ 0.2 is almost as long as that of the MHK model. This is possibly because the appropriate $s_{\text {threshold }}$ increases the communication times of the agent outside the confidence bound and enables the opinion to reach consensus easily. The communication times of agents outside the confidence bound 

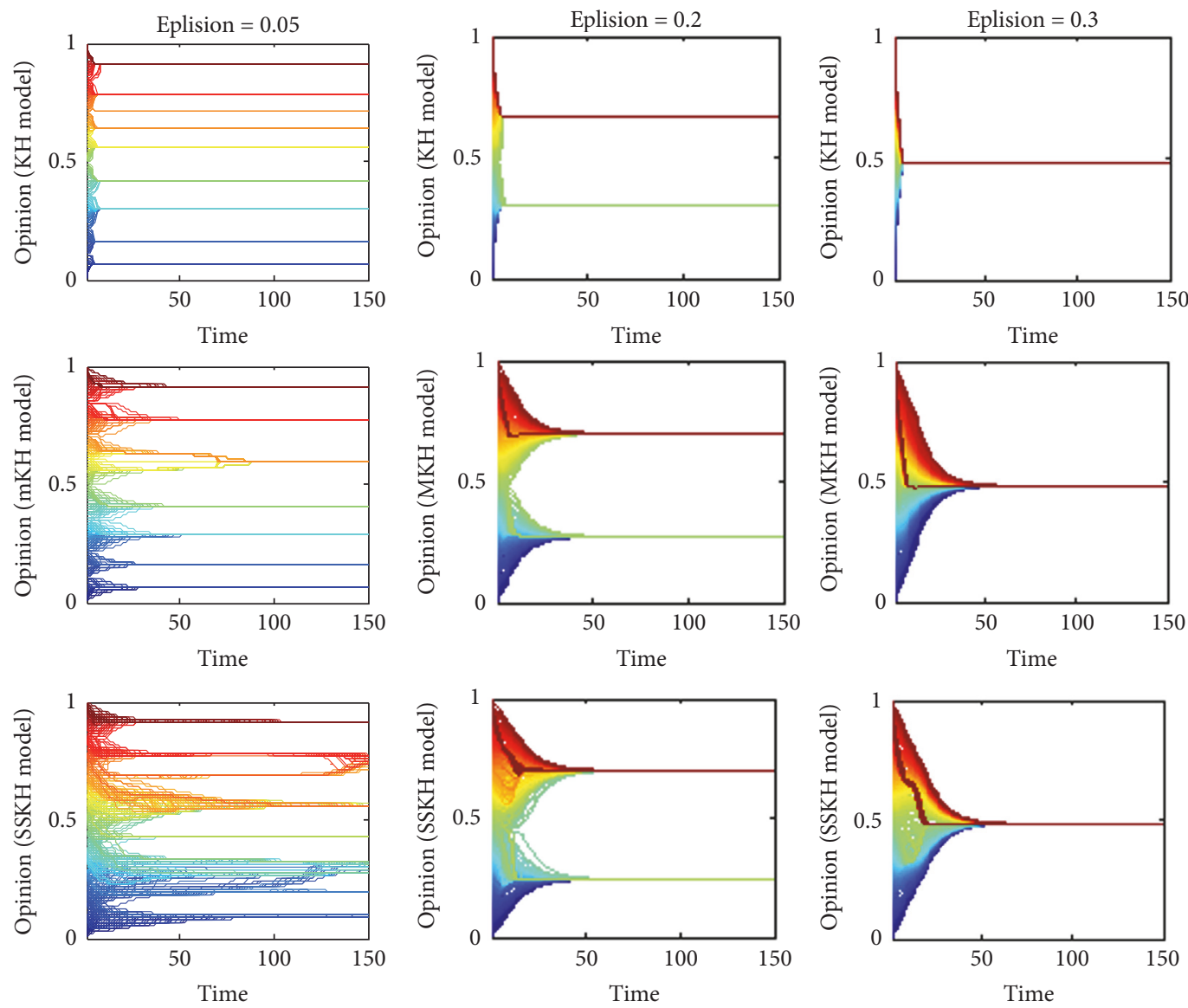

FIGURE 4: Opinion evolution results in the classical HK model, MHK model, and SSHK model with different confidence bounds $\left(w_{s}=0.3\right.$, $s_{\text {threshold }}=0.1$.

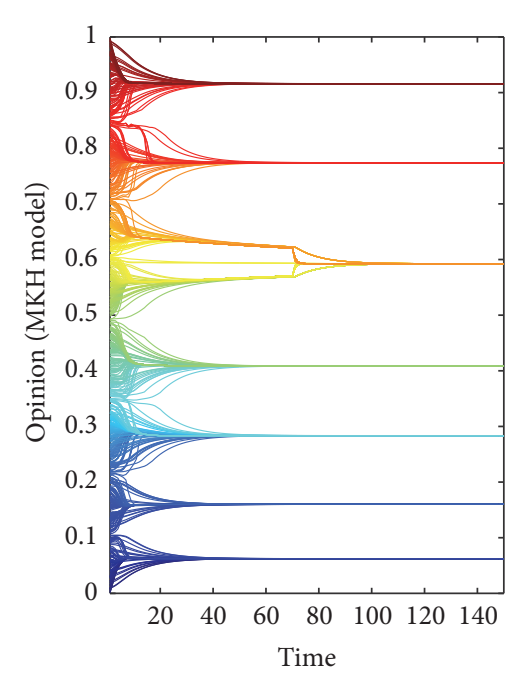

(a)

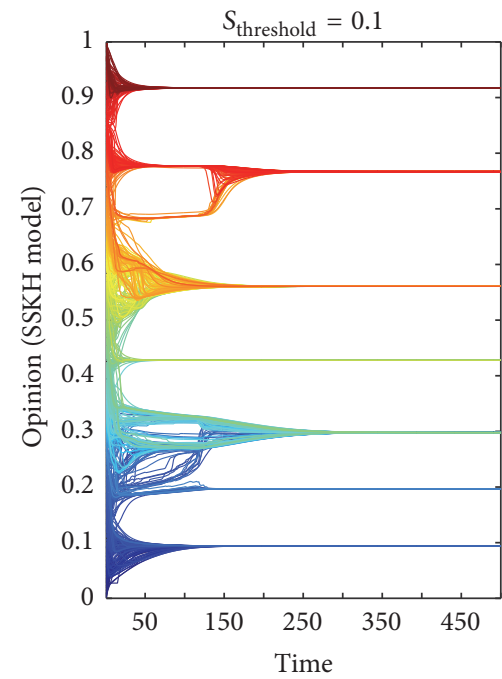

(b)

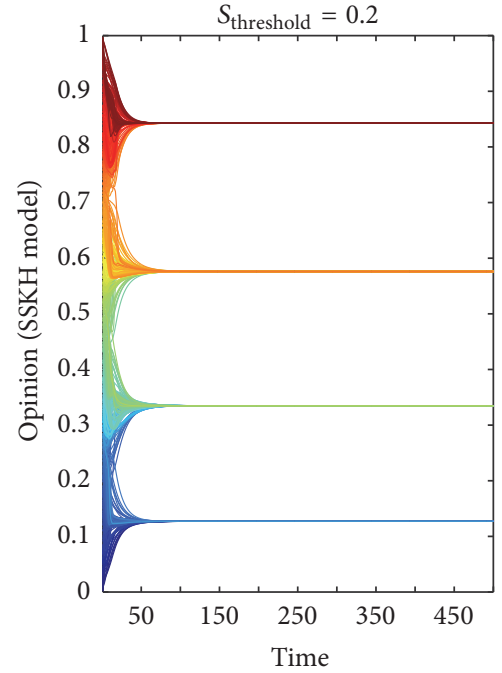

(c)

FIGURE 5: Comparison of opinion evolution results in the MHK model and SSHK model $\left(w_{s}=0.3, s_{\text {threshold }}=0.1,0.2\right)$ when $\varepsilon=0.05$. 
TABLE 1: Comparison of stable time $(T)$, opinion cluster number $(F)$, and the relative size of the largest cluster $N_{\max }$ in the MHK model and SSHK model $\left(w_{s}=0.3\right)$ when $\varepsilon=0.05$.

\begin{tabular}{lccc}
\hline & MHK & $\begin{array}{c}\text { SSHK } \\
s_{\text {threshold }}=0.1\end{array}$ & $\begin{array}{c}\text { SSHK } \\
s_{\text {threshold }}=0.2\end{array}$ \\
\hline$F$ & 7 & 7 & 4 \\
$T$ & 143 & 440 & 155 \\
$N_{\max }$ & 224 & 243 & 274 \\
\hline
\end{tabular}

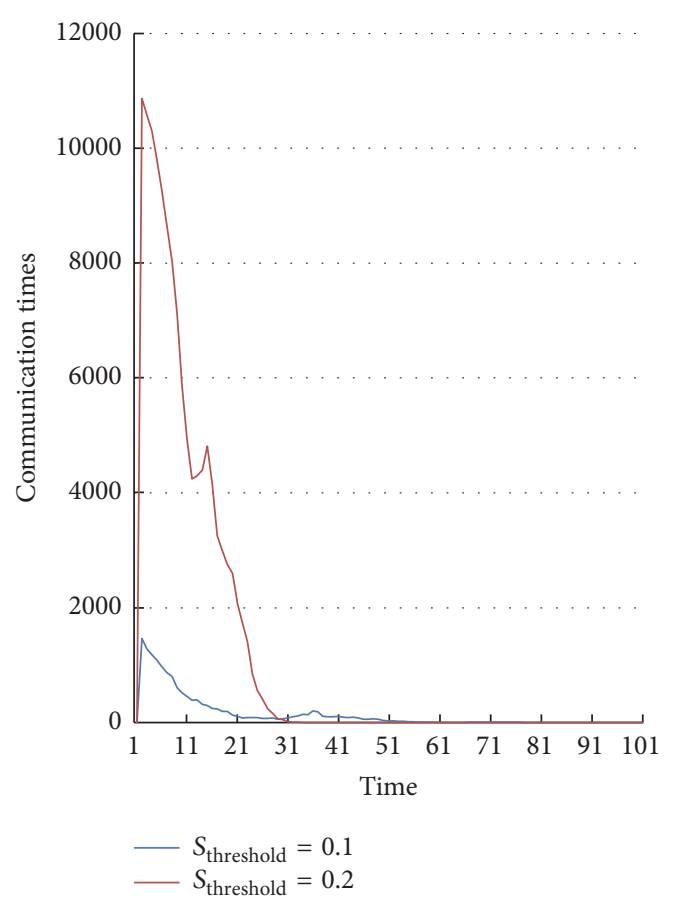

FIgURE 6: Communication times of agents outside the confidence bound in the SSHK model with $s_{\text {threshold }}=0.1$ and $s_{\text {threshold }}=0.2$.

of $s_{\text {threshold }}=0.1$ and $s_{\text {threshold }}=0.2$ are compared in Figure 6 to verify the assumption.

In Figure 6, the communication times increased slightly up to 1700 when $s_{\text {threshold }}=0.1$. When $s_{\text {threshold }}=0.2$, the communication times increased to more than 10000. It can be demonstrated that, as $s_{\text {threshold }}$ increases, the chance of communication between agents outside the confidence bound is improved and the opinion cluster number is decreased to achieve consensus. This result verifies the assumption that the introduction of the social similarity mechanism enables the communication of agents outside the confidence bound and affects opinion evolution.

The communication times of agents outside the confidence bound have significantly decreased and are reduced to 0 after Step 50. This finding can be attributed to opinions that gradually converge to a few clusters with great differences in time and are constrained by the confidence bound to only allow agents with similar opinions to communicate.

3.2. Heterogeneous SSHK Model. Usually, the confidence bound and social similarity threshold of each agent are different. Therefore, the SSHK model of the heterogeneous confidence bound and social similarity threshold is studied in this section. Moreover, the heterogeneous model is then compared with the homogeneous model.

Given that the power-law distribution phenomenon widely exists, the heterogeneous confidence bound and social similarity threshold in this paper follow it. The power-law distribution $p(x)=x^{-a}$ is influenced by the scaling exponent $\alpha$ and minimum value $x_{\min }$ [13].

Each individual may be affected by his neighbors' opinions and social relations. These two effects are different and related. The three relation types are as follows:

(1) The confidence bound and social similarity threshold have a positive correlation. When making a decision, the agent is easily affected by both his neighbors' opinions and their social relationships.

(2) The confidence bound and social similarity threshold have a negative correlation. The psychology of agents is extreme. They change their opinions because of communication with their neighbors or their social relationships.

(3) The confidence bound and social similarity threshold are not correlated. The effects of these factors on agents are random.

In Figure 7, the influence of the above correlation types on opinion evolution is reflected by $F$ (opinion cluster number).

From Figure 7, it can be seen that no correlation shows the most opinion numbers when the confidence bound is small (confidence bound $=0.05$ ), followed by negative correlation, and the opinion numbers of positive correlation are slightly less than those of the negative correlation. Moreover, the opinion numbers of the no correlation type are greater than those of both the positive and negative correlation types. It can be concluded that no correlation obtains the greatest number of opinions regardless of the value of the trust radius and the similarity threshold.

In Figure 8, the influence of the above correlation types on opinion evolution is reflected by $T$ (stable time).

It can be seen from Figure 8 that the effect of correlation between the confidence bound and the similarity threshold on opinion numbers is consistent with the stable time. No correlation requires the longest stable time, followed by negative correlation and positive correlation. Positive and negative correlations reach consensus in the shortest amount of time with no significant difference between them when the confidence bound is small. As the confidence bound increases, it can be seen that the positive correlation type shares exactly the same result as the negative correlation, and the stable times of these two correlation types are much less than that of the no correlation.

Combining the opinion cluster numbers and stable time, it can be concluded that the result obtained by opinion evolution is always the worst in any case when the similarity threshold and the confidence bound are irrelevant; that is, it achieves most opinion cluster numbers and takes the longest time to stabilize. However, as long as there is a correlation between the two factors, whether positive or 

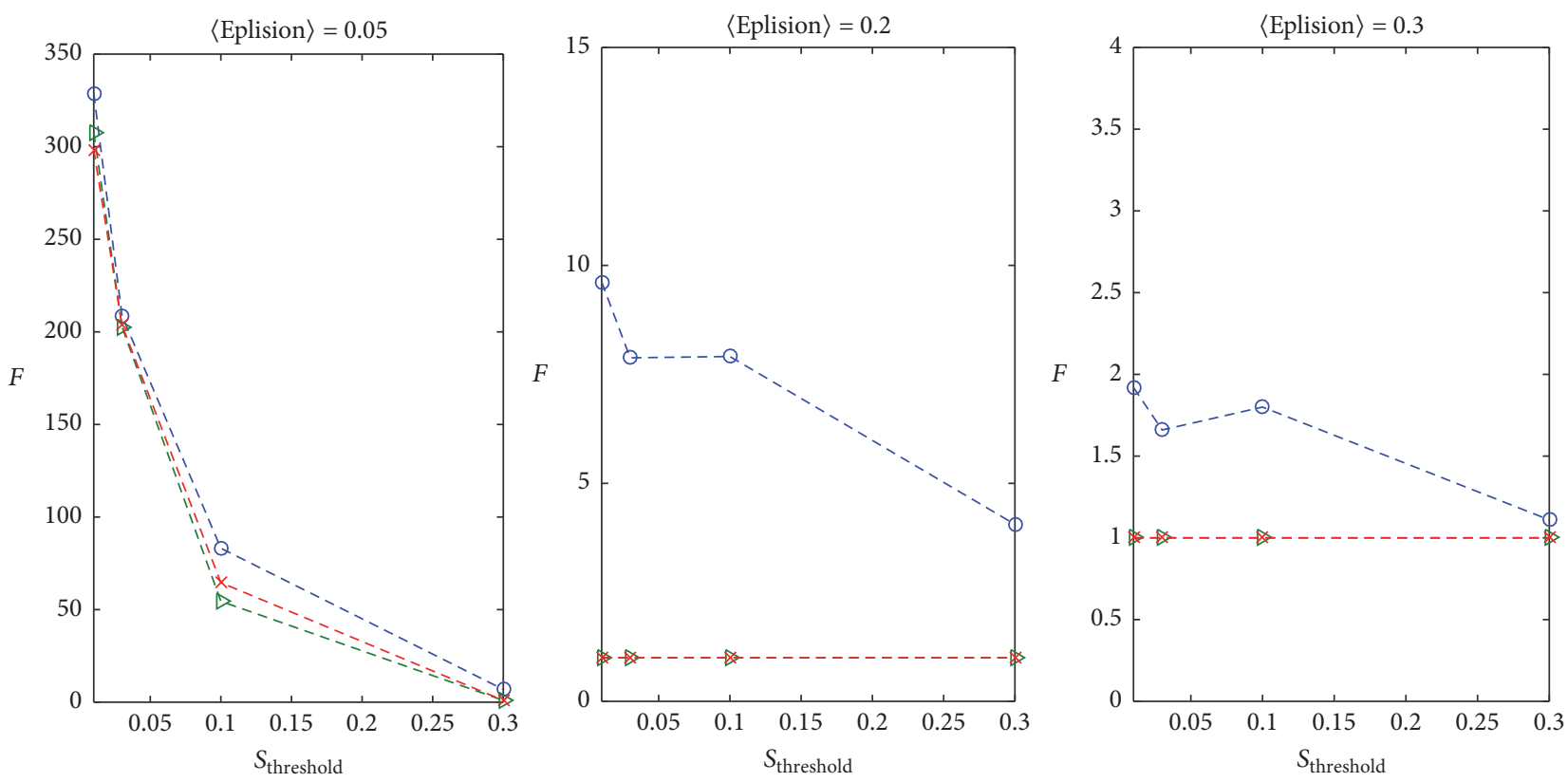

$$
\begin{aligned}
& -\ominus-\text { No correlation } \\
& -\diamond-\text { Positive correlation }
\end{aligned}
$$$$
-\ominus-\text { No correlation }
$$$$
-\diamond \text { - Positive correlation }
$$$$
\text { -*- Negative correlation }
$$

$$
\begin{aligned}
& -\ominus-\text { No correlation } \\
& -\diamond-\text { Positive correlation } \\
& -\star-\text { Negative correlation }
\end{aligned}
$$

FIgURE 7: Comparison of $F$ (opinion cluster number) on the SSHK model under three correlation types of confidence bound and social similarity threshold, wherein $\alpha=3$. Every result is averaged 100 times.
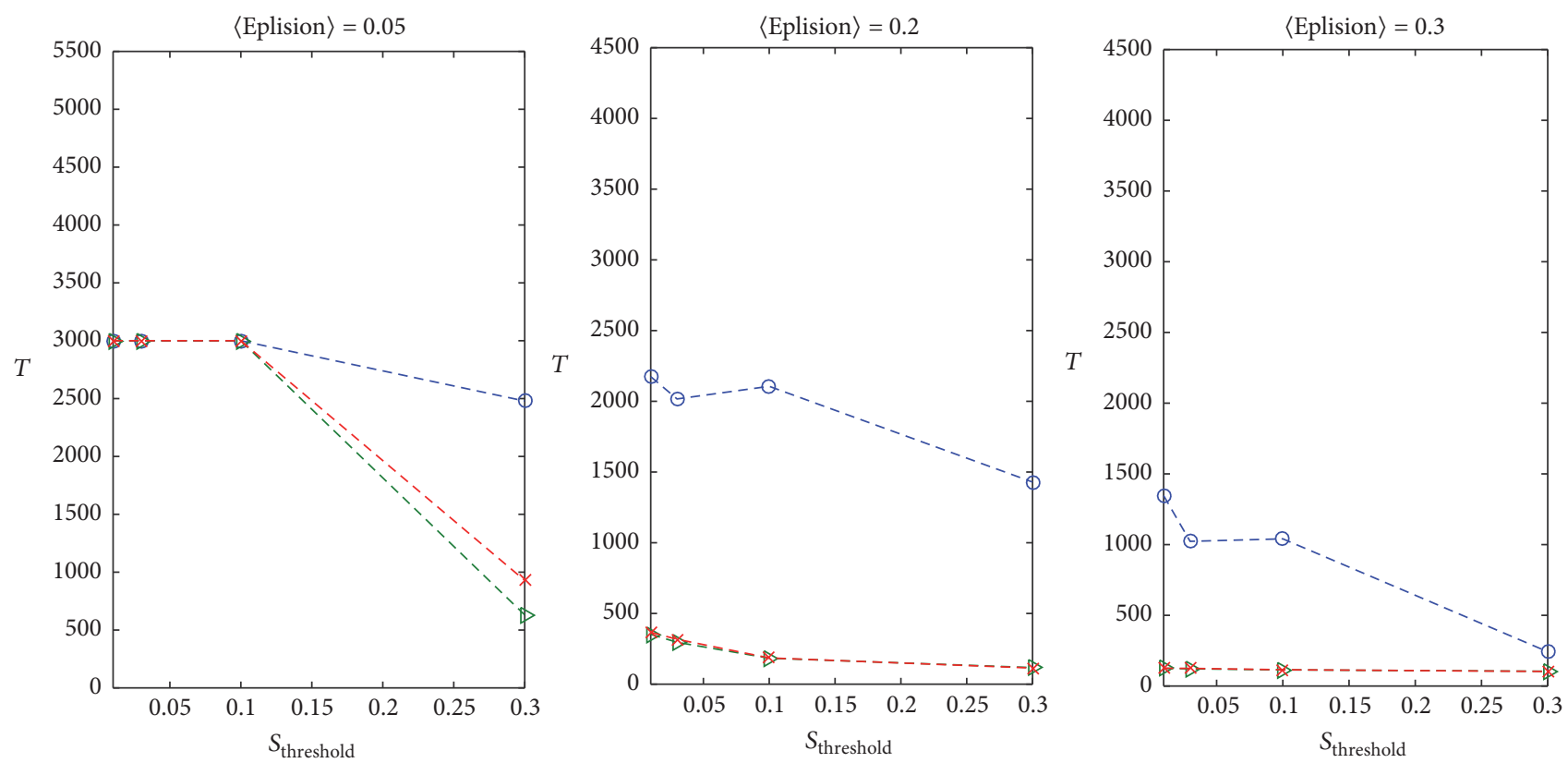

$-\ominus-$ No correlation
$-\diamond-$ Positive correlation
$-*-$ Negative correlation

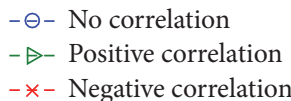

$-\ominus-$ No correlation
$-\diamond-$ Positive correlation
$-\star-$ Negative correlation

FIGURE 8: Comparison of $T$ (stable time) on the SSHK model under three correlation types of confidence bound and social similarity threshold, wherein $\alpha=3$. Every result is averaged 100 times. 


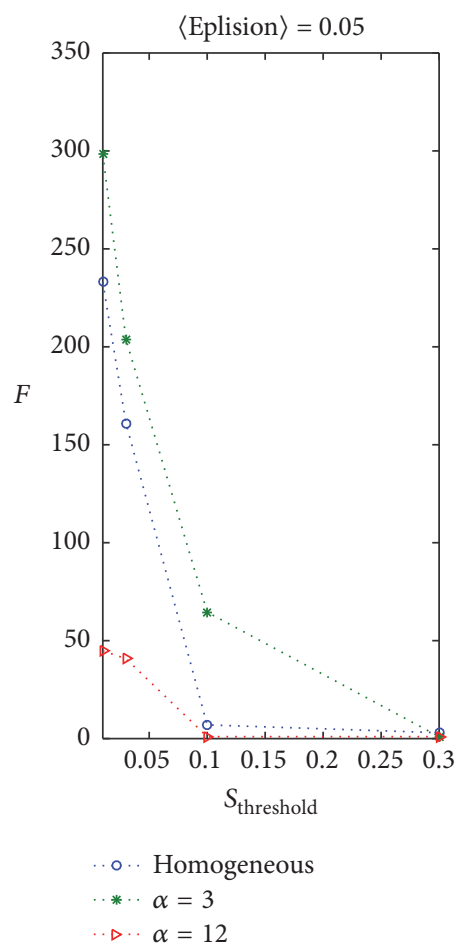

(a)

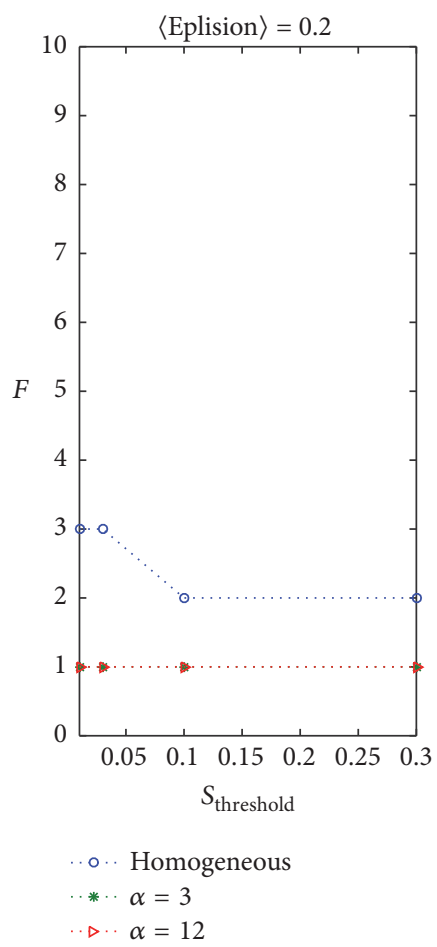

(b)

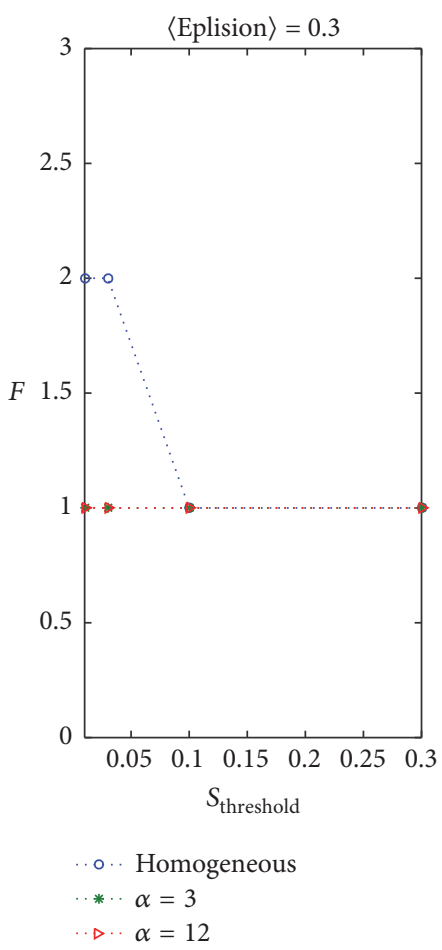

(c)

FIGURE 9: Comparison of $F$ (opinion cluster number) on the heterogeneous SSHK model and homogeneous SSHK model with the negative correlation between the confidence bound and social similarity threshold. Every result is averaged 100 times.

negative correlation, the results are not significantly different. The results are the same when the confidence bound is not small.

The homogeneous and heterogeneous models are now compared. The heterogeneous model ( $\alpha=3$ and $\alpha=12$ ) represents different heterogeneity. A small scaling exponent corresponds to a distribution that is more heterogeneous. Given that the correlation between the confidence bound and social similarity threshold may affect the opinion convergence in the study, no correlation and negative correlation are selected in the heterogeneous model.

First, the homogeneous model is compared with the heterogeneous model with negative correlation between the confidence bound and social similarity threshold. The result is shown in Figure 9.

Figure 9(a) shows that when the scaling exponent is small $(\alpha=3, \varepsilon=0.05)$, the final opinion cluster number of the heterogeneous SSHK model is more than that of the homogeneous model, whereas the opinion number of the heterogeneous SSHK model $(\alpha=12, \varepsilon=0.05)$ is less than that of the homogeneous model. Therefore, heterogeneity may not always promote opinion consensus compared to a homogeneous model. When the confidence bound increase is not small $(\varepsilon=0.2$ and $\varepsilon=0.3)$, the heterogeneous model always facilitates opinion convergence, whereas the homogeneous model cannot. It can be concluded that the heterogeneous model obtains a better opinion consensus result than the homogeneous model, except when the scaling exponent and confidence bound are small enough.
The homogeneous model is now compared with the heterogeneous model of no correlation in Figure 10.

In Figures 10(a) and 10(b), when the confidence bound is small, the model with the smaller scaling exponent $(\alpha=3)$ obtains more opinion cluster numbers than the homogeneous model. However, the homogeneous model shows a larger $F$ value than that in the model with a larger scaling exponent $(\alpha=12)$. Figure 9 (c) shows the approximate result.

In conclusion, compared with the homogeneous model, the heterogeneous model can lead to decreasing opinion cluster numbers and promote the opinion to achieve consensus. This finding is related to the distribution of the confidence bound and social similarity threshold as well as their correlations.

\section{Conclusion}

This paper proposed an improved SSHK model based on a classical bounded confidence model by introducing social similarity between agents into the MHK model, simultaneously considering the influence of neighbors' opinions and their social relationships as well. The SSHK model considers the special situation that a small number of agents outside the confidence bound have the chance to exchange opinions. The homogeneous and heterogeneous SSHK models are also studied and compared in this paper. The result shows that the new model also obtains the main features of the opinion result, namely, fragmentation, polarization, and consensus. 


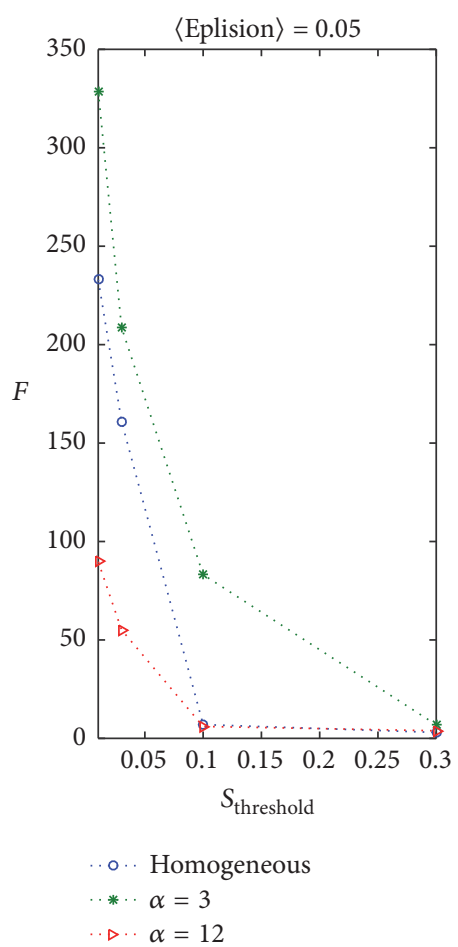

(a)

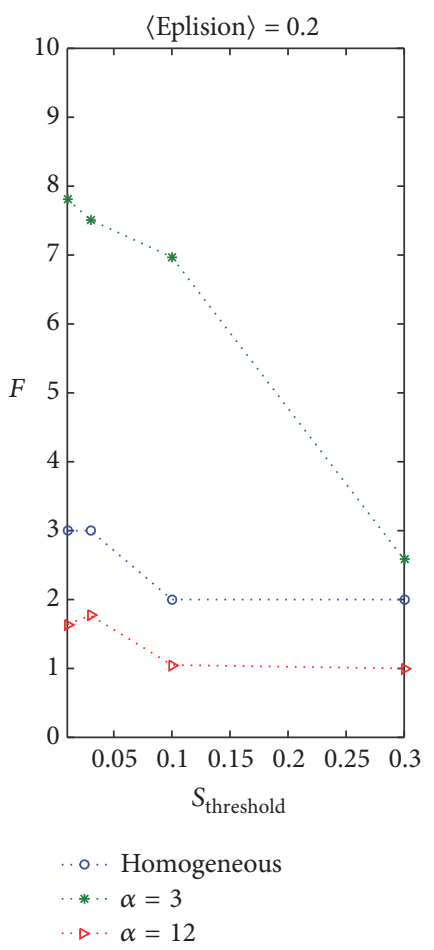

(b)

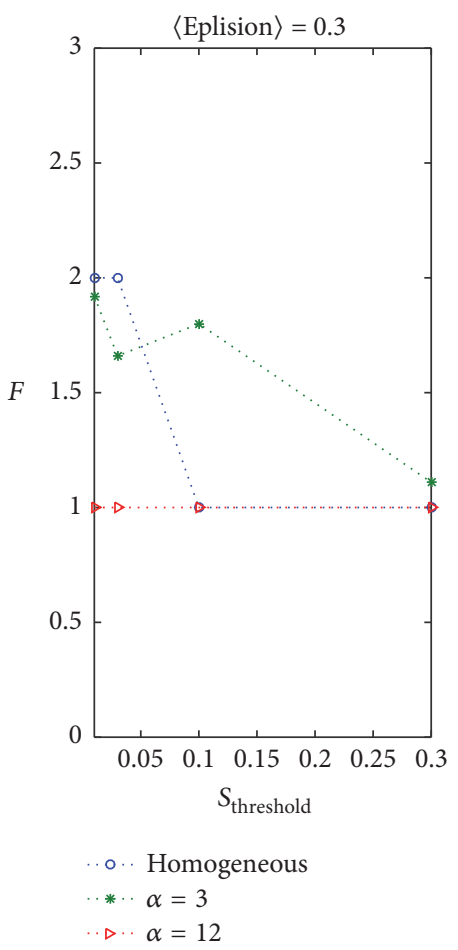

(c)

FIGURE 10: Comparison of $F$ (opinion cluster number) on the heterogeneous SSHK model and homogeneous SSHK model with no correlation between the confidence bound and social similarity threshold. Every result is averaged 100 times.

By studying the homogeneous SSHK model, it can be concluded that the new model can easily achieve consensus under an appropriate social similarity threshold compared to the classical models; that is, social similarity also influences opinion dynamics [15]. The increase in the social similarity threshold changes the opinion from fragmentation to consensus. Moreover, the decrease in social similarity threshold hardly enables the opinion to reach consensus. When the influences of the confidence bound and social similarity are compared, the former shows a stronger effect on opinion convergence than the latter. Further, the comparison result of the SSHK model with the HK and MHK models reveals that the SSHK model can more easily reach consensus, because agents outside the confidence bound can communicate and exchange opinions.

The heterogeneous model with the influence of correlation between the confidence bound and social similarity threshold is discussed and compared with the homogeneous model. Compared to the homogeneous model, the heterogeneous model promotes the opinion to reach consensus. When the confidence bound is related to social similarity threshold, the heterogeneous model enables the opinion to converge easily, especially when the confidence bound and social similarity threshold are positively correlated. This finding suggests that opinion consensus is easily reached when individuals treat the influence of neighbors' opinions and social relationships as equal. Opinion is easier to converge when all individuals are in extreme conditions; that is, they are only affected by neighbors' opinions, or they are only influenced by social relationships. However, opinion is difficult to predict and reaching consensus is difficult when there is no correlation. Thus, the correlation between the confidence bound and social similarity threshold influences the state of opinion stability. This finding may provide a theoretical basis and method for the control or guidance of public opinion. Some agents with a positive or negative correlation between similarity threshold and the confidence bound may join and lead the community to reach an agreement.

In summary, regardless of the nature of the results (i.e., reality or experimental), social similarity between individuals also influences the opinion evolution result. The impact of social relationships between individuals on opinion dynamics cannot be ignored. Introducing the social relationship and social attributes of individuals into the opinion evolution model makes the model more realistic. Moreover, the introduction of these concepts provides a theoretical framework for the opinion dynamics model, which considers complex individual social attributes and relationships. This study ignores the influence of network topology. The influence of complex social networks and heterogeneous individual social attributes on public opinion evolution needs further research.

\section{Conflicts of Interest}

The authors declare that there are no conflicts of interest regarding the publication of this article. 


\section{Acknowledgments}

This work was supported by the National Natural Science Foundation of China (Grant no. 71571081, no. 61540032, and no. 91324203).

\section{References}

[1] J. Li and R. Xiao, "Agent-based modelling approach for multidimensional opinion polarization in collective behaviour," Journal of Artificial Societies and Social Simulation, vol. 20, no. 2, article 4, 2017.

[2] R. Xiao, J. Hou, and J. Li, “Dynamic evolution of government's public trust in online collective behaviour: A social computing approach," International Journal of Bio-Inspired Computation, vol. 9, no. 1, pp. 1-18, 2017.

[3] T. Lux, "Rational forecasts or social opinion dynamics? Identification of interaction effects in a business climate survey," Journal of Economic Behavior \& Organization, vol. 72, no. 2, pp. 638655, 2009.

[4] R. Xiao and T. Yu, "A multi-agent simulation approach to rumor spread in virtual commnunity based on social network," Intelligent Automation and Soft Computing, vol. 17, no. 7, pp. 859-869, 2011.

[5] G. Deffuant, D. Neau, F. Amblard, and G. Weisbuch, "Mixing beliefs among interacting agents," Advances in Complex Systems (ACS), vol. 3, pp. 87-98, 2000.

[6] R. Hegselmann and U. Krause, "Opinion dynamics and bounded confidence: models, analysis and simulation," Journal of Artificial Societies and Social Simulation, vol. 5, pp. 3-33, 2002.

[7] P. Liu and X. Chen, "An overview on opinion spreading model," Journal of Applied Mathematics and Physics, vol. 3, no. 4, pp. 449-454, 2015.

[8] S. Fortunato, "On the consensus threshold for the opinion dynamics of Krause-Hegselmann," International Journal of Modern Physics C, vol. 16, no. 2, pp. 259-270, 2005.

[9] W. Zhang, M.-S. He, and R. Jin, "Opinion dynamics in complex networks," Lithuanian Journal of Physics, vol. 53, no. 4, pp. 185194, 2013.

[10] M. Jalili, "Social power and opinion formation in complex networks," Physica A: Statistical Mechanics and its Applications, vol. 392, no. 4, pp. 959-966, 2013.

[11] M. E. J. Newman, "Power laws, Pareto distributions and Zipf's law," Contemporary Physiscs, vol. 46, no. 5, pp. 323-351, 2005.

[12] J. Lorenz, "A stabilization theorem for dynamics of continuous opinions," Physica A: Statistical Mechanics and its Applications, vol. 355, no. 1, pp. 217-223, 2005.

[13] H. Liang, Y. Yang, and X. Wang, "Opinion dynamics in networks with heterogeneous confidence and influence," Physica A: Statistical Mechanics and its Applications, vol. 392, no. 9, pp. 2248-2256, 2013.

[14] G. Fu, W. Zhang, and Z. Li, "Opinion dynamics of modified Hegselmann-Krause model in a group-based population with heterogeneous bounded confidence," Physica A: Statistical Mechanics and its Applications, vol. 419, pp. 558-565, 2015.

[15] L. Guo and X. Cai, "Continuous opinion dynamics in complex networks," Computer Physics Communications, vol. 5, pp. 10451053, 2009.

[16] J. Lorenz, "Heterogeneous bounds of confidence: meet, discuss and find consensus!," Complexity, vol. 15, no. 4, pp. 43-52, 2010.
[17] P. Nyczka, "A model of opinion dynamics with bounded confidence and noise," https://arxiv.org/abs/1106.0008.

[18] S. Chen, D. H. Glass, and M. McCartney, "Characteristics of successful opinion leaders in a bounded confidence model," Physica A: Statistical Mechanics and its Applications, vol. 449, pp. 426-436, 2016.

[19] Q. Liu and X. Wang, "Opinion dynamics with similarity-based random neighbors," Scientific Reports, vol. 3, no. 10, article 2968, 2013.

[20] H. Wang and L. Shang, "Opinion dynamics in networks with common-neighbors-based connections," Physica A: Statistical Mechanics and its Applications, vol. 421, pp. 180-186, 2015.

[21] A. Flache and M. Mäs, "How to get the timing right. A computational model of the effects of the timing of contacts on team cohesion in demographically diverse teams," Computational and Mathematical Organization Theory, vol. 14, no. 1, pp. 23-51, 2008.

[22] M. Mäs, A. Flache, K. Takács, and K. A. Jehn, "In the short term we divide, in the long term we unite: Demographic crisscrossing and the effects of faultlines on subgroup polarization," Organization Science, vol. 24, no. 3, pp. 716-736, 2013.

[23] A. Flache, M. Mäs, T. Feliciani et al., "Models of social influence: towards the next frontiers," Journal of Artificial Societies and Social Simulation, vol. 20, no. 4, 2017.

[24] X. Chen, L. Zhang, and W. Li, "A network evolution model for chinese traditional acquaintance networks," IEEE Intelligent Systems, vol. 29, no. 5, pp. 5-13, 2014.

[25] M. Pineda and G. M. Buendía, "Mass media and heterogeneous bounds of confidence in continuous opinion dynamics," Physica A: Statistical Mechanics and its Applications, vol. 420, pp. 73-84, 2015. 


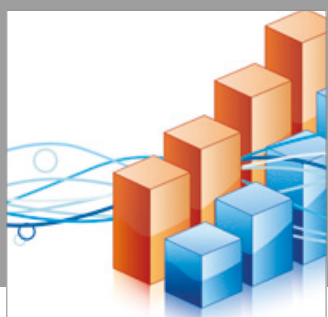

Advances in

Operations Research

vatersals

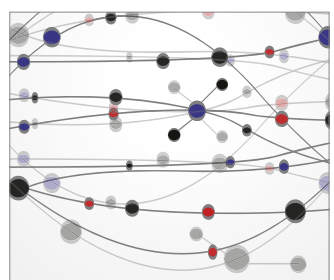

\section{The Scientific} World Journal
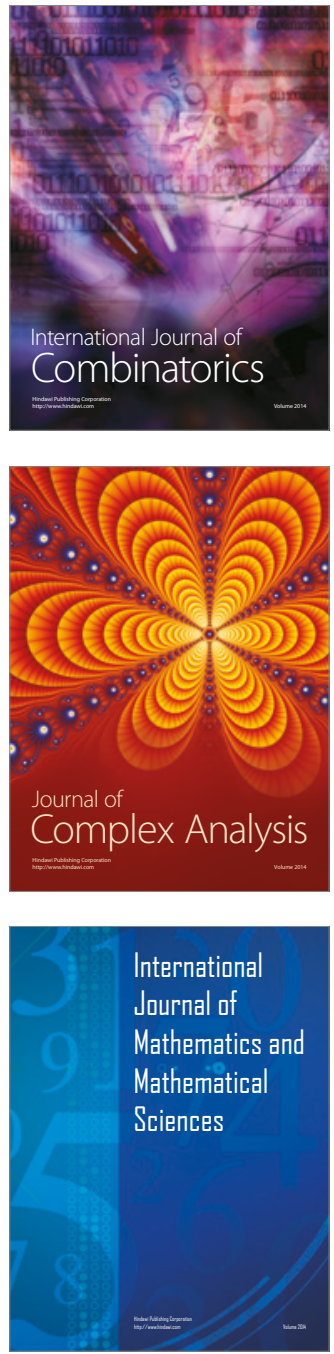
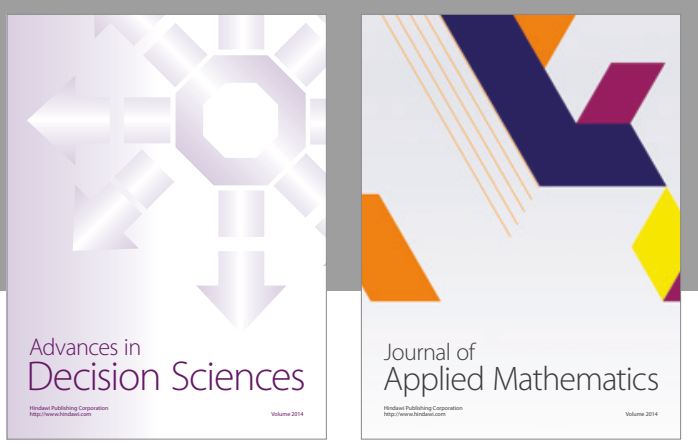

Algebra

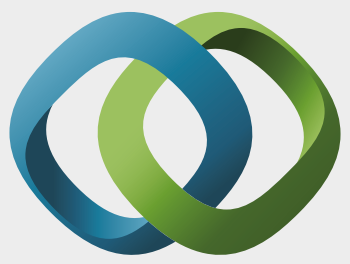

\section{Hindawi}

Submit your manuscripts at

https://www.hindawi.com
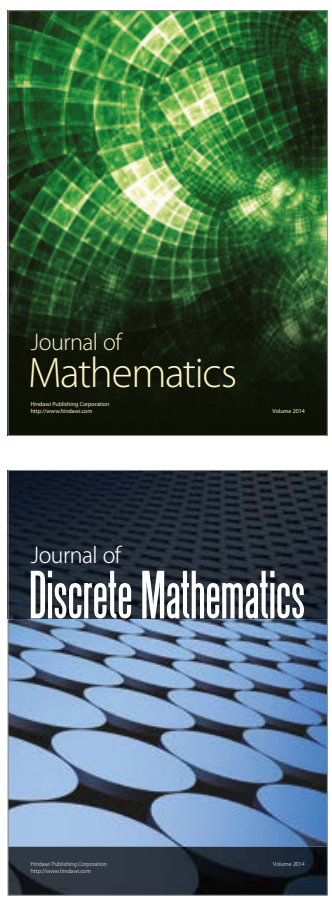

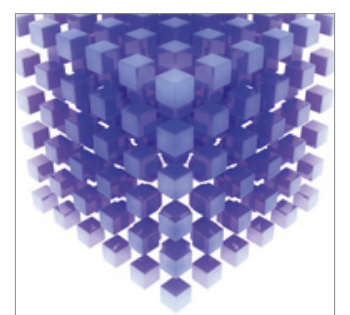

Mathematical Problems in Engineering
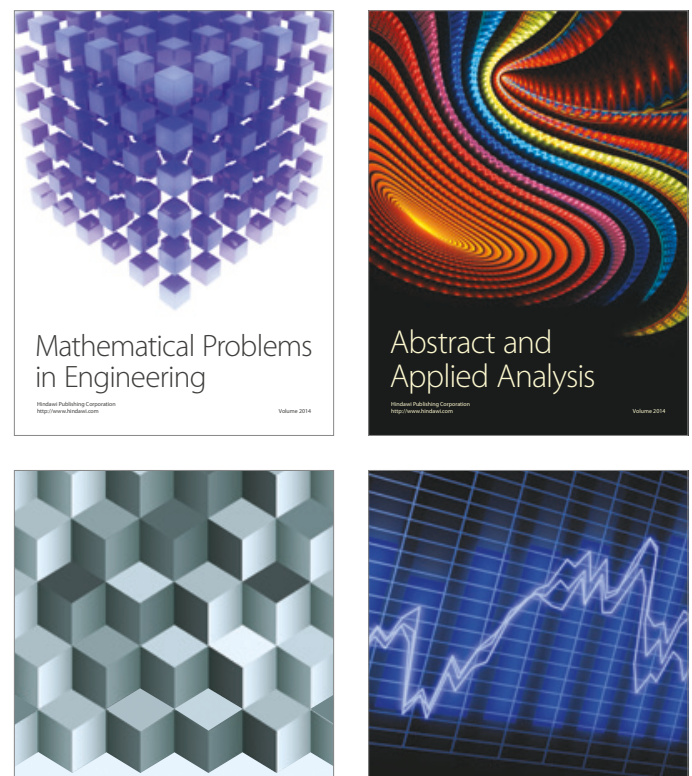

Journal of

Function Spaces

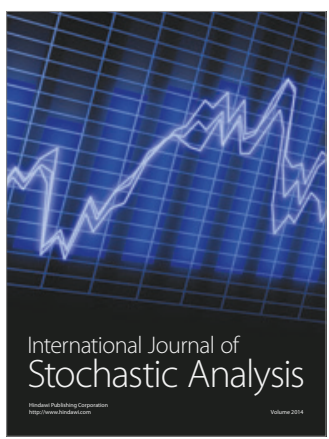

Probability and Statistics
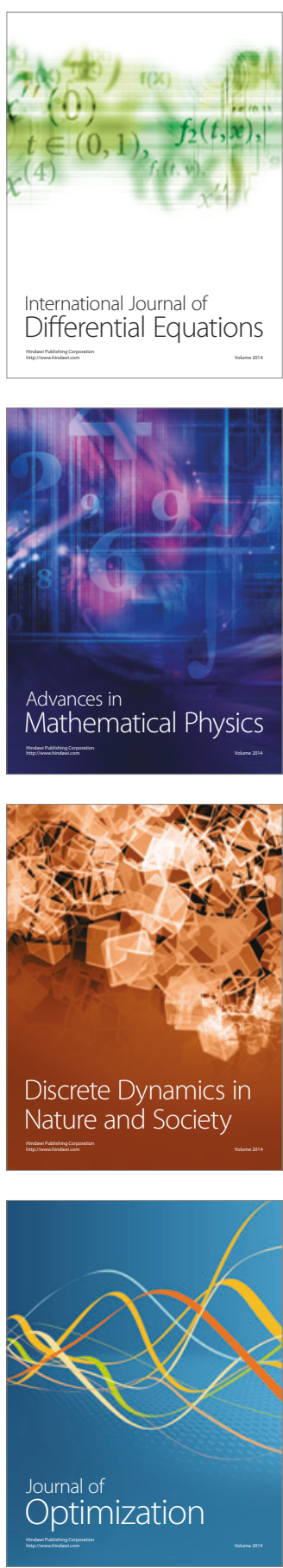